\title{
Sehr geehrte Damen und Herren, liebe DGLRM-Mitglieder,
}

ereignisreiche Tage liegen hinter uns, gerade bin ich von der 53. Jahrestagung unserer Gesellschaft am Forschungsflughafen in Braunschweig zurück. Noch sind die Eindrücke ganz frisch.

\section{DGLRM-Tagung in Braunschweig}

Nach allem was wir in den vergangenen Tagen gelernt haben, hätte es kaum einen besseren Ort als den Forschungsflughafen Braunschweig für unsere Tagung geben können. Das verkehrsträgerübergreifende Portfolio, das diesen Flughafen zu Europas größtem Kompetenzzentrum für Mobilitätsfragen und Verkehrstechnik sowie zu einem der innovativsten Wirtschafts- und Wissenschaftscluster in Europa gemacht hat, haben wir sehr anschaulich vorgeführt bekommen. Insofern bin ich dem Hausherrn, Herrn Professor Joachim Block, dem Leiter der DLR-Standorte Braunschweig und Göttingen auch sehr dankbar, dass er uns die Gelegenheit geben hat, unsere Tagung in Räumen des DLR auszurichten. Auch war ich von der Selbstverständlichkeit und Offenheit, mit der wir dort empfangen wurden, außerordentlich beeindruckt und möchte mich dafür im Namen aller Tagungsteilnehmer sehr herzlich bedanken. Mein besonderer Dank gilt vor allem Frau Carola Meyer und Frau Julia Goertner von der Forschungsflughafen Braunschweig GmbH sowie Herrn Mark Schmidt, dem Verantwortlichen für die Standortentwicklung und Kommunikation des DLR in Braunschweig. Sie haben uns hier in allen Fragen kompetent beraten und unterstützt und mit großem Engagement die Fachbesichtigungen und auch den Gesellschaftsabend arrangiert und vorbereitet. Damit haben Sie einen großen Anteil daran, dass diese Tagung für uns alle zu einem besonderen Erlebnis geworden ist.

\section{Vielfältiges wissenschaftliches Programm}

Das Programm war sehr vielgestaltig, die Vorträge gehaltvoll und interessant - insgesamt ergab sich ein wunderbares Mosaik des multidisziplinären Fachs Flugmedizin. Die vielseitigen Diskussionen haben gezeigt, dass das Spektrum der
Themen dem entsprach, was die in der Luft- und Raumfahrtmedizin tätigen Kollegen tagtäglich bewegt. Ich denke, ein Jeder von uns konnte daraus etwas für sich mitnehmen. Die Veranstaltung ist von der Landesärztekammer Niedersachsen in der Kategorie B für das „Fortbildungszertifikat der Ärztekammer" mit insgesamt 12 Fortbildungspunkten und vom Luftfahrtbundesamt als flugmedizinischer Fortbildungslehrgang gemäß MED.D.030 der EU-Verordnung 1178/2011 mit 11 Stunden anerkannt worden.

Die beiden Plenarvorträge von Bernd Jeuken und Hannes Ross haben uns in die Welt der Luftfahrt geführt und uns Einblicke in Themen gegeben, die uns Luftund Raumfahrtmedizinern nicht so geläufig sind. In jedem Fall aber haben sie dabei ganz besondere Akzente gesetzt. Erneut konnten wir Posterpreise und auch den Albrecht-Ludwig-BerblingerWissenschaftspreis 2015 der Deutschen Akademie für Flug- und Reisemedizin gGmbH verleihen.

\section{Preisträger 2015}

Die Posterpreisträger 2015 sind:

1. Backhaus C, Homann H, Felten C, Hedtmann J, Jäger M

Entwicklung eines Hilfsmittels zur Unterstützung mobilitätseingeschränkter Personen in Verkehrsflugzeugen

2. Werner A, Jakobs FM, Kreutzmann U, Frischmuth J

Einflüsse der Hypoxie auf die Farbdiskriminierung zur Qualifizierung und Quantifizierung des BiV-Sehens mit den modernen night vision goggles

3. Ledderhos C, Gammel C, Gens A Künstliche Schwerkraft als Herausforderung für die Luft- und Raumfahrtmedizin

Der Albrecht-Ludwig-Berblinger-Wissenschaftspreis der Deutschen Akademie für Flug- und Reisemedizin ging in diesem Jahr an Herrn PD Dr. Philipp Zanger aus Heidelberg. Seine Arbeit „Effectivness of Rifaximin in prevention of diarrhoea in individuals travelling to south and southeast Asia", publiziert in der Novemberausgabe 2013 der Zeitschrift „Lancet Infectious Disesases“, die den beeindru-

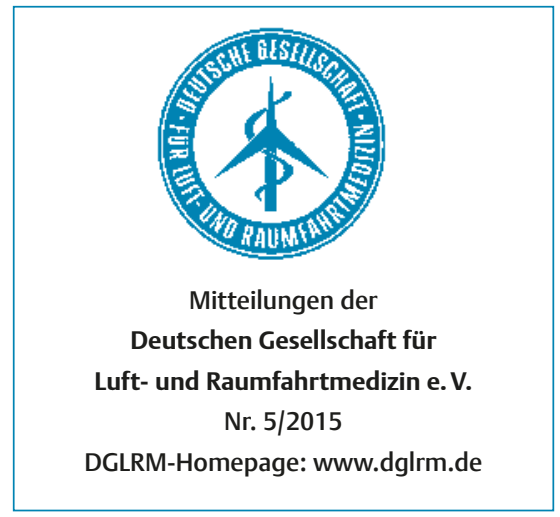

Geschäftsstelle der DGLRM

Christine Gens

Postfach 870204

13162 Berlin

E-Mail: christine.gens@dglrm.de

Tel.: 0176/5624-2876

\section{Wir gratulieren}

- 65 Jahre, Dr. Erich Wahoff, Lingen, 23.10.1950

- 70 Jahre, Dr. Wolfgang Koester, Oberteuringen, 04.10.1945

- 70 Jahre, Prof. Dr. Hans Pongratz, München, 26.10.1945

- 75 Jahre, Dr. Ursula Broll von Horn, Steinhagen, 05.10.1940

Wir begrüßen als neue Mitglieder

- Dr. Andreas Grove, Sinzheim

- PD Dr. Martin Kammerl, Zwiesel

- Roland Stepan, Fulda

- Kai Stukenbrock, Schwelm

- Dr. Christian Königer, Neubiberg

- Marian Venus-Aengenheister, Pfäffikon, Schweiz

- Dr. Franziska Grube, Fassberg

- Julius Sander, Münster 


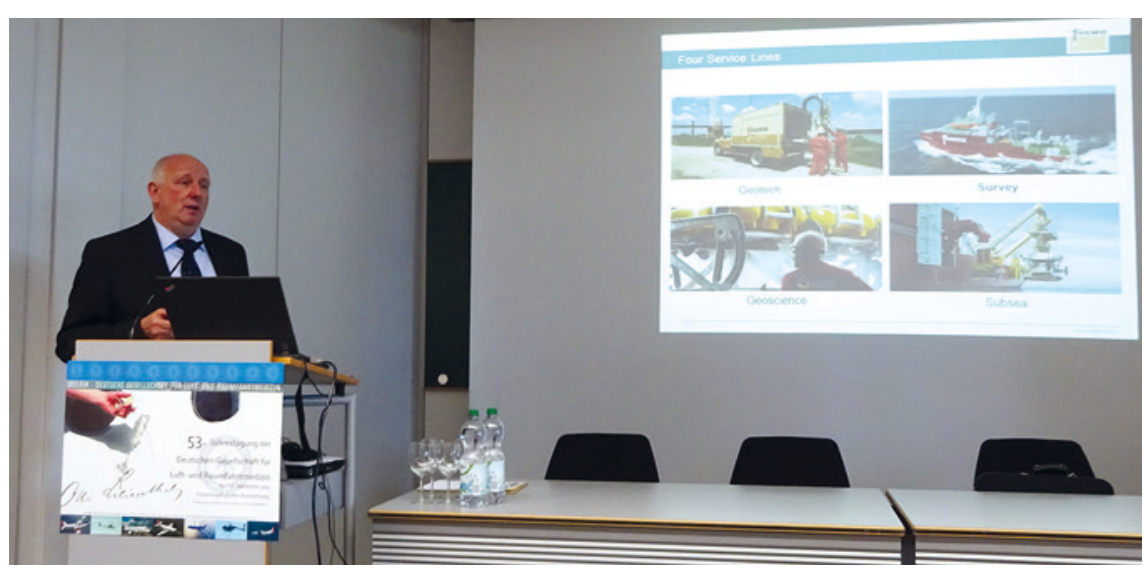

Abb. 1 Bernd Jeuken bei seinem Plenarvortrag.

Quelle: PD Dr. Jochen Hinkelbein

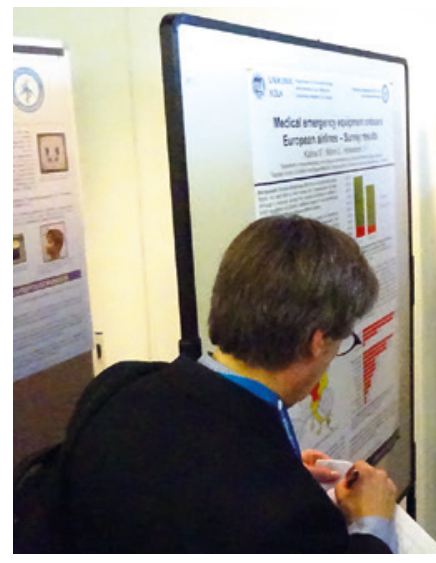

Abb. 2 Posterdiskussion.

ckenden Impact Factor von 19,45 hat, konnte auch die Jury überzeugen und wurde als beste der eingereichten Arbeiten ausgewählt.

\section{Fachbesichtigungen}

Die verschiedenen Fachbesichtigungen bei der Bundesstelle für Flugunfalluntersuchung (BFU), beim Deutschen Zentrum für Luft- und Raumfahrt (DLR) sowie beim Niedersächsischen Forschungszentrum für Luftfahrt (NFL) und bei der Aerodata AG am Freitagnachmittag haben uns die Vielfalt der Themen, die hier vor Ort in Braunschweig am Forschungsflughafen zum Thema Mobilität bearbeitet werden, zumindest andeutungsweise vor Augen geführt. Gerne hätten viele von uns noch mehr erfahren.

\section{Mitgliederversammlung}

Wie gewohnt, gab es auf der Mitgliederversammlung die Rechenschaftslegung des Vorstands und der Arbeitsgruppenleiter über die im letzten Jahr geleistete Arbeit. Im Mittelpunkt aber standen Diskussionen zum Budget der Gesellschaft und zur Zukunft der Deutschen
Akademie für Flug-und Reisemedizin (DAF).

Die letzte Beitragserhöhung wurde im Jahr 2007 beschlossen und, wie damals, müssen wir inzwischen feststellen, dass der derzeitige Beitrag von 100 Euro nicht mehr ausreicht, um eine angemessene wissenschaftliche Arbeit zu leisten und insbesondere unseren Nachwuchs gebührend zu fördern. Auch die wissenschaftliche Jahrestagung leidet regelmäßig unter zu geringen Finanzmitteln und das Einsparpotenzial ist inzwischen ausgeschöpft, wenn man nicht an die für die Gesellschaft existenziellen Säulen, wie sie beispielsweise die Zeitschrift eine darstellt, gehen möchte. Nach ausführlicher Diskussion sind die anwesenden Mitglieder übereingekommen, dass wir auf der nächsten Mitgliederversammlung einen Punkt „Beitragsänderung“ in die Tagesordnung aufnehmen müssen.

Im Hinblick auf die zivile Aus- und Fortbildung der Flugmediziner sieht die DGLRM die unbedingte Notwendigkeit, diese zu erhalten. Sie hat auch konkrete Vorschläge dazu unterbreitet, jedoch drängt inzwischen die Zeit. Noch vor Erscheinen dieser Ausgabe wird es weiterführende Gespräche mit der Lufthansa zur Zukunft der DAF geben, von denen wir uns eine trag- und zukunftsfähige Lösung erhoffen. Wir werden Sie in diesem Punkt auf dem Laufenden halten.

\section{Podiumsdiskussion}

Die Podiumsdiskussion am Sonnabendnachmittag zum Thema „Quo vadis Flugmedizin“ hat die Verantwortlichen der Flugmedizin in Deutschland an einem Tisch zusammengeführt. Leider war die Zeit viel zu kurz, um die angesprochenen Themen tiefgründiger zu behandeln. Dennoch ist hiermit der Grundstein gelegt worden, um mit den Verantwortlichen im BMVI und dem Luftfahrtbundesamt sowie dem Luftfahrtamt der Bundeswehr zukünftig in engere Kontakte einzutreten.

\section{Rahmenprogramm}

Das Rahmenprogramm gab uns die Möglichkeit auch einen Hauch von der Schönheit der Stadt Braunschweig zu erhaschen. Der Nachtwächter „Hugo“ hat es verstanden, uns die Besonderheiten und

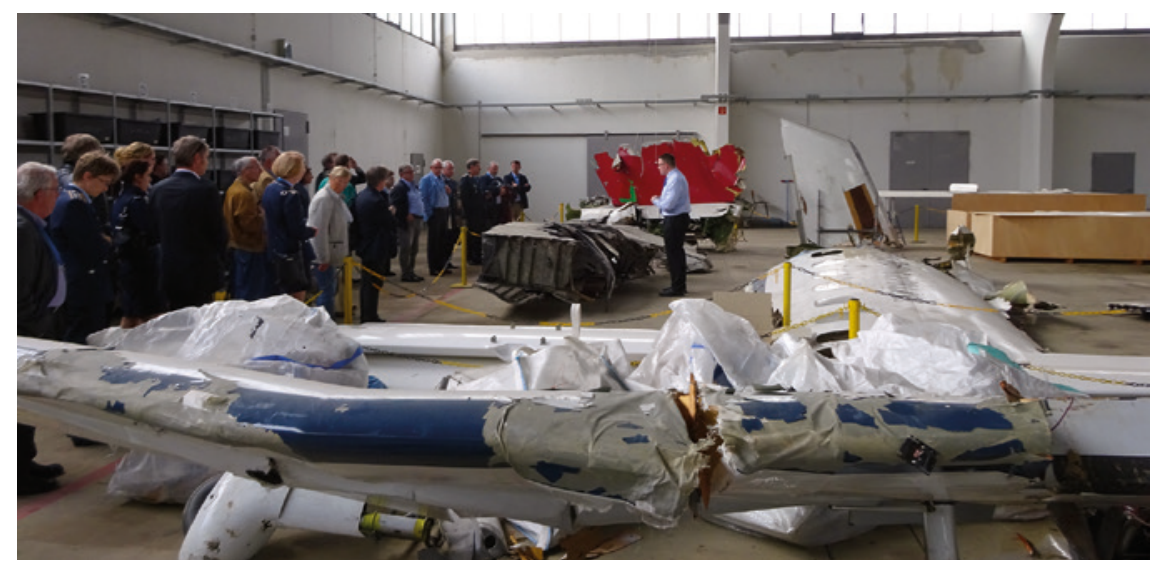

Abb. 3 Teilnehmer der Tagung im Hangar der BFU. 


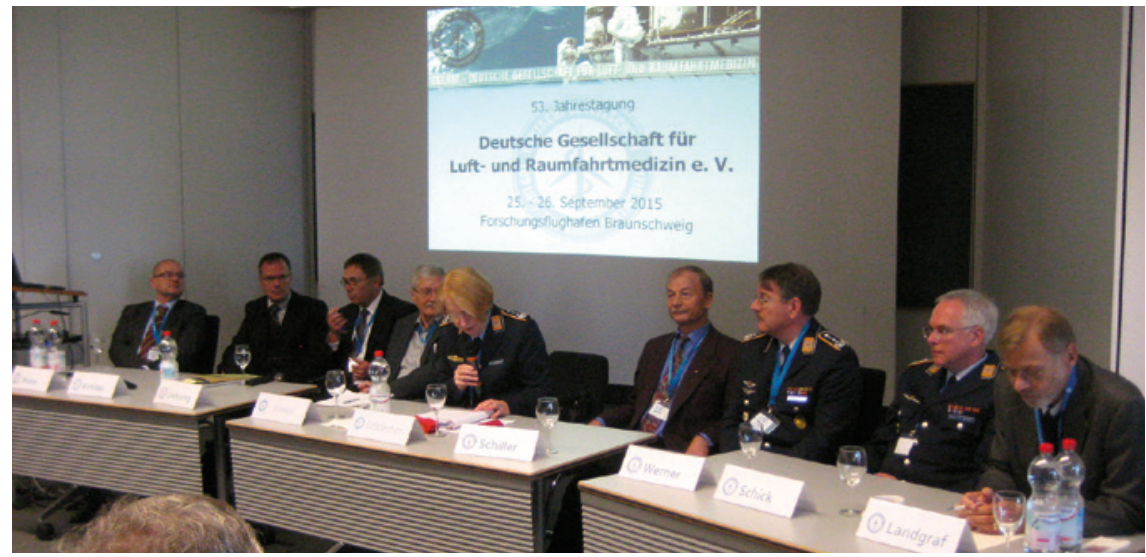

Abb. 4 Podiumsdiskussion am Sonnabendnachmittag.

Quelle: Prof. Dr. Hans Pongratz

Geschichte der Stadt sehr kurzweilig zu vermitteln. Der Gesellschaftsabend in der „Dornse“, diesem wunderbaren Saal im alten Rathaus, zu dem die Stadt Braunschweig die Teilnehmer der Tagung zu einem Empfang eingeladen hat, stellte einen ganz besonderen Höhepunkt zum Abschluss der Tagung dar. In ihrer ambitionierten Begrüßungsrede hat die Bürgermeisterin der Stadt, Frau Cornelia RohsePaul, alle Tagungsteilnehmer von der Besonderheit der Region um Braunschweig überzeugen können. Ganz sicher werden uns diese Tage in Braunschweig in guter Erinnerung bleiben und den einen oder anderen sicher zu einem „Zweitbesuch“ anregen.

\section{Erfolgreiche Tagung durch gute}

Zusammenarbeit

An dieser Stelle möchte ich es nicht versäumen, mich ganz herzlich bei allen Kolleginnen und Kollegen zu bedanken, die mit ihrem Engagement zum Gelingen der Tagung beigetragen haben. Dies schließt die Vortragenden genauso ein, wie die Sitzungsleiter, die Mitglieder des Vorstands sowie die des Vorstandsrats. Nur durch diese gute Zusammenarbeit war eine erfolgreiche Durchführung der Tagung möglich. Mein ganz besonderer Dank geht dabei an das Organisationsteam um Frau Christine Gens und Herrn Frank Teichert, das mit dieser Tagung seine Feuertaufe bestanden hat. Bei Herrn Andreas Pongratz möchte ich mich für die Abwicklung der gesamten Tagungsanmeldungen und die schnelle Informationsgenerierung zur Tagung über unsere Homepage bedanken.

\section{DGLRM-Tagung 2016}

Ohne die Aktivitäten ihrer Mitglieder kommt eine Gesellschaft nicht aus. Ich

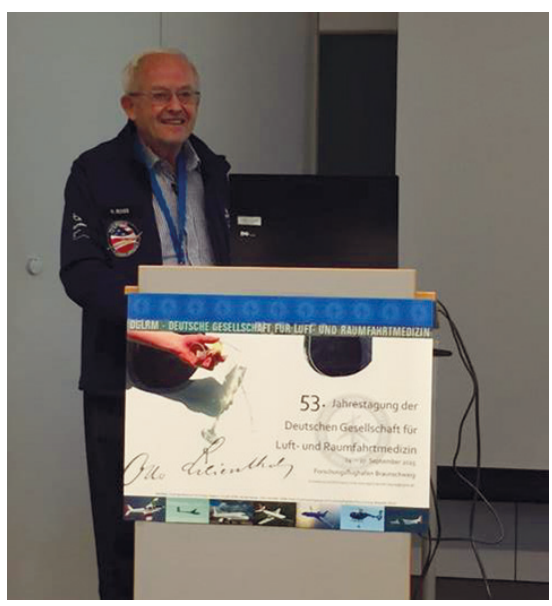
Quelle: Dr. Ilse Janicke

möchte Sie daher auch im nächsten Jahr zu einer aktiven, konstruktiven Teilnahme am Leben der Gesellschaft ermuntern. Ihre Beiträge für unsere Zeitschrift und/oder die Homepage sind uns stets willkommen. Bitte nehmen Sie auch den 31.03.2016 als Deadline für das Einreichen der Vortrags- und Posterbeiträge zu unserer nächsten, der 54. Jahrestagung, die vom 22. bis 24. September im Hubschraubermuseum in Bückeburg stattfinden wird, schon in Ihren Kalender auf. Nähere Informationen dazu erhalten Sie in der folgenden Ausgabe der FTR und auf unserer Homepage.

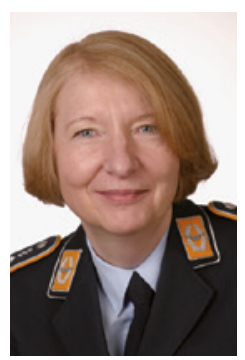

Abb. 5 Hannes Ross als Plenarredner.
Lehrgänge 2015

23. Refresher-Seminar Flugmedizin (deutsch)

Dt. Akademie für Flug- und Reisemedizin $\mathrm{GmbH}$

Termin: 06.11.-08.11.2015

Ort: $\quad$ Lufthansa Training \& Conference Center, Seeheim-Jugenheim

Kontakt: Sigrid Froese, Tel: +49 (0)69/69691222

Internet: www.flugmed.org/ www.eusam.org

EUSAM - Basic course 26 (englisch) European School of Aviation Medicine Termin: 03.09.-11.09.2016

Ort: Medizinischer Dienst der Deutschen Lufthansa auf der Lufthansa-Basis, Flughafen Frankfurt

Kontakt: Sigrid Froese, Tel: +49(0)69/69691222

Internet: www.flugmed.org/ www.eusam.org

EUSAM - Advanced course 26 (englisch) European School of Aviation Medicine

Termin: 03.12.-11.12.2016

Ort: Medizinischer Dienst der Deutschen Lufthansa auf der Lufthansa-Basis, Flughafen Frankfurt

Kontakt: Sigrid Froese, Tel: +49 (0)69/69691222

Internet: www.flugmed.org/ www.eusam.org

\section{Verantwortlich für den Inhalt} der DGLRM-Seiten

Deutsche Gesellschaft für Luft- und Raumfahrtmedizin e.V. (DGLRM) OFA PD Dr. Carla Ledderhos, Präsidentin Zentrum für Luft- und Raumfahrtmedizin der Luftwaffe

Str. der Luftwaffe 322, 82242 Fürstenfeldbruck CarlaLedderhos@bundeswehr.org

Bankverbindung

Deutsche Bank Hamburg

Konto-Nr.: 388948200

BLZ: 20070000

IBAN: DE 63200700240388948200 BIC: DEUTDEDBHAM

Änderungen für die Mitgliederkartei bitte an: Dipl. Ing. Christine Gammel

Zentrum für Luft- und Raumfahrtmedizin der Luftwaffe

Str. der Luftwaffe 322

82242 Fürstenfeldbruck

ChristineGammel@bundeswehr.org 